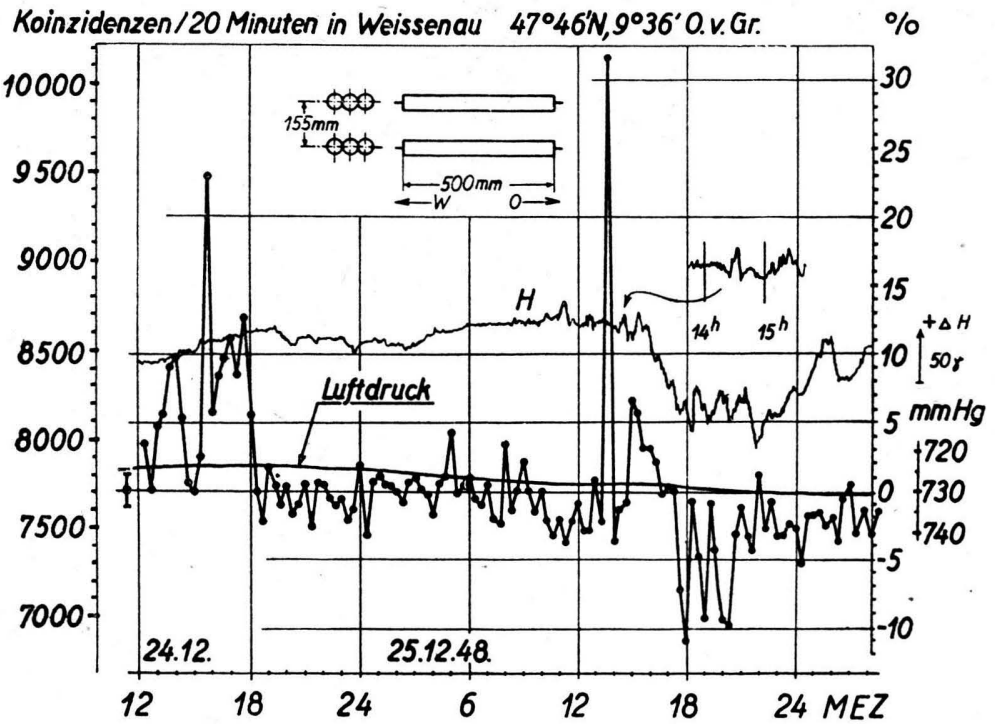

bzw. $\mathrm{D}_{2} \mathrm{O}$-Moleküls handeln würde, wäre zu erwarten, daß bei einer Mischung von $\mathrm{H}_{2} \mathrm{O}$ und $\mathrm{D}_{2} \mathrm{O}$ als zusätzliches Spektrum das des HDO-Moleküls in Erscheinung treten müßte. Da aber die Mischung nur eine Überlagerung der beiden erwähnten Spektren zeigt und kein drittes Spektrum, so können als Träger dieser Spektren nur das 2-atomige $\mathrm{OH}, \mathrm{OH}^{+}$ode $\mathrm{OH}^{-}$, bzw. OD, $\mathrm{OD}+$ oder OD- in Frage kommen.

In einer früheren Untersuchung ${ }^{1}$ wurd ein Spektrum beschrieben, das bei Glimmentladungen in $\mathrm{H}_{2} \mathrm{O}$-Dampf in der positi ven Säule auftritt. Es liegt im Sichtbaren und zeigt eine sehr einfache Rotationsstruktur (nur P- und R-Zweig) (Abb. 1. Spektrum a). In Erweiterung dieser Versuche wurde nun das $\mathrm{D}_{2} \mathrm{O}$ untersucht, und im gleichen Spektralbereich ein Spektrum von ähnlicher Einfachheit der Rotationsstruktur gefunden (Abb. 1, Spektrum b). Es sind 10 Banden, die nach Rot abschattiert sind, und deren Kanten folgende Lage haben:

$14.30 \mathrm{~h} \mathrm{MEZ} \mathrm{am} \mathrm{25.} \mathrm{Dez.} 1948$ ist von der seltener Art, die $\mathrm{How} \mathrm{e}^{2}$ und $\mathrm{Bartels}, \mathrm{Heck}$ und $\mathrm{J}$ oh n s t o $n^{3}$ näher beschrieben haben. Der Störungsvektor hat dabei eine andere Richtung als derjenige der Baystörungen bei Mögel-Dellinger-Effekten. Der Verauf dieser Störung ist in der Abb. nochmals in vergrößertem Zeitmaßstab herausgezeichnet. In der Registrierung des Fraunhofer-Institutes bei Freiburg erscheint sie prägnanter als in Fürstenfeldbruck und in Wingst. Ferner ist ein Zusammenhang zwischen den merkwürdig hohen Schwankungen der Teilchenzahl zwischen $17 \mathrm{~h}$ und $21 \mathrm{~h}$ am 25. Dez. 1948 und den magnetischen Pulsationen während des magnetischen Sturmes angedeutet. Er ist durch die statistischen Schwankungen verzerrt und kann aus diesem einen Beispiel nicht einwandfrei ermittelt werden. Bei der bisher üblichen einstündigen Registrierung wurden solche Schwankungen ausgemittelt!

Den HHrn. Professoren Bartels, Burkhar t, Errulat und $\mathrm{Ki}$ e penhe e r danke ich für die freundliche Übermittlung von erdmagnetischem Registriermaterial bzw. von Sonnenaufnahmen.

2 H. H. How e, Terrestr. Magnetism 44, 339 [1939].

3 J. B a r tels, N. H. He ck und H. F. Joh nst o $n$, Terrestr. Magnetism 44, 411 [1939].

\section{Über ein neues OD-Spektrum im Sichtbaren}

Von H. S c h ü l e r und L. R e i n e be ck

Kaiser-Wilhelm-Institut für Physik, Max-Planck-Institut, Hechingen

(Z. Naturforschg. 4 a, 560-561 [1949]; eingeg. am 17. Sept. 1949)

Es wird ein Spektrum beschrieben, das in der Glimmentladung mit $\mathrm{D}_{2} \mathrm{O}$-Dampf beobachtet wird. Das Spektrum liegt im Sichtbaren und stellt das Analogon dar zu dem früher in $\mathrm{H}_{2} \mathrm{O}$-Dampf gefundenen Spektrum. Wenn es sich hierbei um Spektren des $\mathrm{H}_{2} \mathrm{O}$ -

$\begin{array}{rccl}\text { Band } 1 & \lambda 5475 \mathrm{AE} & \boldsymbol{v}=18260 \mathrm{~cm}^{-1} \text { (Bei den Wellen- } \\ 2 & 5209 & 19192 & \text { längenangaben } \\ 3 & 4923 & 20307 & \text { ist mit einem } \\ 4 & 4794 & 20854 & \text { Fehler von } \\ 5 & 4631 & 21588 & \pm 1 \mathrm{AE} \text { zu rech- } \\ 6 & 4517 & 22132 & \text { nen) } \\ 7 & 4444 & 22496 & \\ 8 & 4344 & 23014 & \\ 9 & 4243 & 23562 & \\ 10 & 4180 & 23917 & \end{array}$

Diese Banden lassen sich in ein Termschema ein ordnen (Abb. 2). Man sieht, daß im unteren Zustand die Differenzenfolge $\Delta v=1426,1281,1115,932 \mathrm{~cm}^{-1}$ und im angeregten Zustand $\Delta v=546,357 \mathrm{~cm}^{-1}$ auftreten. Die Einfachheit der Struktur dieser Banden zeigt Abb. 3. Während in Bande $3 \mathrm{P}$ - und $\mathrm{R}-\mathrm{Zweig}$ sich so überdecken, daß man praktisch nur eine einzige Linienfolge sieht, läßt Bande 2 bei höheren Rotationen beide $\mathrm{Zweige}$ getrennt erscheinen. Auf der Abb. 3 kann man 18-19 Rotationslinien jeder Bande erkennen.

Bezüglich des Trägers dieses Spektrums sei be merkt, daß es sich um Beobachtungen mit Hilfe der früher beschriebenen Entladungsröhre ${ }^{2}$ in der positiven Säule einer Glimmentladung handelt in reinem strömendem $\mathrm{D}_{2} \mathrm{O}$-Dampf ohne Zusatz von Fremdgasen. Als Träger dieses Leuchtens kommt zunächst da: 3-atomige $\mathrm{D}_{2} \mathrm{O}$-Molekül in Frage. Zur Prüfung dieser Annahme ging man von folgender Utberlegung aus: Das $\mathrm{H}_{2} \mathrm{O}$ zeigt ja, wie oben erwähnt, ein entsprechendes Spektrum. Wenn diese beiden analogen Spektren

1 H. S chüler u. A. Wo eld i ke, Physik. Z. 44, 335 [1943].

${ }^{2}$ H. S c h ü l e r u. A. Wo el d i ke, Physik. Z. 
dem $\mathrm{D}_{2} \mathrm{O}$ bzw. $\mathrm{H}_{2} \mathrm{O}$ zuzuschreiben wären, dann sollte man bei einer Mischung von $\mathrm{H}_{2} \mathrm{O}$ und $\mathrm{D}_{2} \mathrm{O}$ ein neues drittes Spektrum des HDO in dem gleichen Gebiet erwarten. Versuche, die mit einer Mischung von gleichen Teilen $\mathrm{H}_{2} \mathrm{O}$ und $\mathrm{D}_{2} \mathrm{O}$ durchgeführt wurden, ergaben nun ein Spektrum, das sich eindeutig als Überlagerung der im $\mathrm{D}_{2} \mathrm{O}$ und der im $\mathrm{H}_{2} \mathrm{O}$ erhaltenen Banden herausstellte. Ein neues zusätzliches Spektrum (des HDO) trat nicht in Erscheinung.

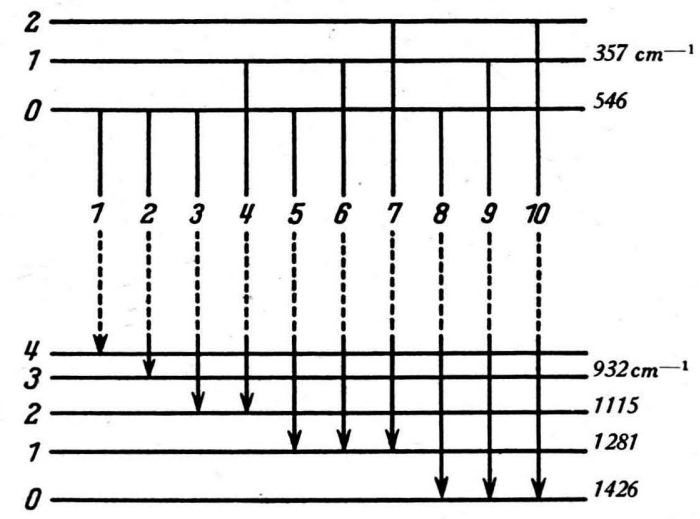

Abb. 2. Termschema OD.

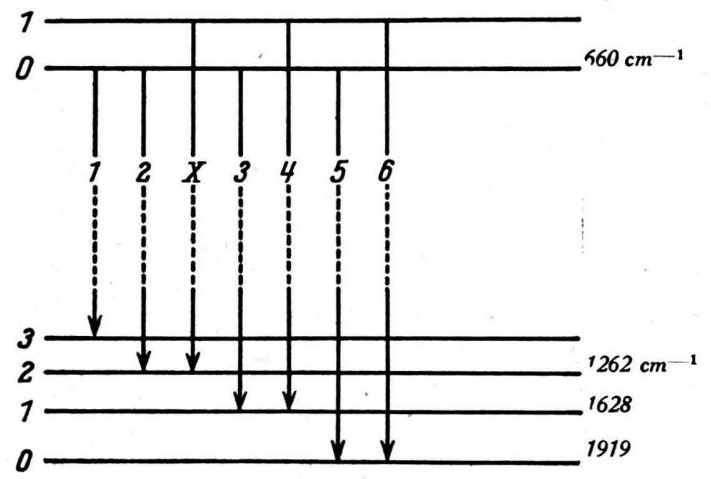

Abb. 4. Termschema $\mathrm{OH}$.

Damit ist bewiesen, daß es sich nicht um das Leuchten des $\mathrm{H}_{2} \mathrm{O}$ - bzw. $\mathrm{D}_{2} \mathrm{O}$-Moleküls handelt; und gleichzeitig wird die Schwierigkeit behoben, die auftritt, wenn man dieses Spektrum mit seiner einfachen Struktur wie in der früheren Arbeit ${ }^{1}$ als einen Übergang zwischen höher angeregten Termen des 3-atomigen Wassermoleküls zu deuten versucht. Während man damals beim $\mathrm{H}_{2} \mathrm{O}$ die intensivste Bande als (0-0)-Bande ansprach, kommt man nun zu einer anderen Einordnung im Hinblick auf die Befunde beim reinen $\mathrm{D}_{2} \mathrm{O}$, bei dem sich ja das Termschema durch die zahlreicheren Banden bestimmter festlegen läßt. Danach ergibt sich für das im $\mathrm{H}_{2} \mathrm{O}$-Dampf beobachtete Spektrum das Termschema der Abb. 4; auf diese Einordnungsmöglichkeit hat uns Hr. Her z ber g bereits freundlicherweise aufmerksam gemacht.

Diese Einordnung hat nun insofern eine Bestätigung gefunden, als eine weitere Bande, die das
Schema fordert, nachgewiesen wurde. Diese Bande (hier mit $\mathrm{x}$ bezeichnet) ist sehr schwach. Sie konnte erst beobachtet werden, nachdem es gelungen war, die Intensität des Leuchtens um etwa den Faktor 5 zu steigern.

Für den unteren Zustand ergibt sich die Differenzenfolge: $\Delta v=1919,1628,1262 \mathrm{~cm}^{-1}$, während im angeregten Zustand nur zwei Schwingungszustände mit $\Delta v=660 \mathrm{~cm}^{-1}$ auftreten. Die Kanten der 7 Banden haben folgende Wellenlängen (vgl. ${ }^{1}$ ):

$\begin{array}{rcc}\text { Bande } 1 & \lambda 5480,2 \mathrm{AE} & v=18243 \mathrm{~cm}^{-1} \\ 2 & 5125,6 & 19505 \\ \mathrm{x} & 4957 & 20168 \\ 3 & 4730,3 & 21134 \\ 4 & 4587,3 & 21793 \\ 5 & 4336,6 & 23053 \\ 6 & 4216,4 & 23710\end{array}$

Wenn man nach dem Vorhergesagten nun als Träger dieser Spektren nicht mehr 3-atomige Moleküle annehmen darf, so bleibt nur noch übrig, ein 2-atomiges Bruchstück dafür verantwortlich zu machen, also $\mathrm{OH}, \mathrm{OH}^{+}$oder $\mathrm{OH}-$, bzw. OD, OD+ oder OD-. Für einen 2-atomigen Träger sprechen auch die einfachen Termschemata mit nur einem Schwingungstyp.

Nimmt man zunächst $\mathrm{OH}$ oder $\mathrm{OD}$ an, dann stellt man aus den beobachteten Schwingungsdifferenzen fest, daß in beiden Fällen Anfangs- und Endzustand nicht mit den bekannten Zuständen des $\mathrm{OH}$ bzw. OD identisch sind. Da es bisher nicht gelungen ist, außer dem im Ultraviolett liegenden OH-Spektrum (Übergang ${ }^{2} \Sigma \rightarrow{ }^{2} \Pi$ ) eine weitere Emission festzustellen, die den Anschluß zwischen diesen neuen Termen und den bekannten des $\mathrm{OH}$ bzw. des OD herstellt, so ist eine Deutung dieser Spektren als neutrale OH- bzw. OD-Spektren nicht sehr wahrscheinlich. Es steht also. mehr $\mathrm{OH}^{+}, \mathrm{OH}_{-}$bzw. OD + , OD- zur Diskussion. Versuche, aus den Aufspaltungen der höheren Rotationslinien Aufschluß über die Multiplizität zu erhalten, haben mit den bisherigen Hilfsmitteln noch zu keinem Ergebnis geführt.

Es sei noch darauf hingewiesen, daß die (0-0)-Bande bei beiden Spektren nicht die intensivste ist, und daß die Hauptintensität der Rotationslinien innerhalb einer Bande bei den ersten Rotationen liegt. Vielleicht sind diese Tatsachen durch die Entstehung der angeregten Bruchstücke bedingt.

\section{Der Hall-Effekt von Rhodium*}

Von P. O. Ge h 1 h of f und E. Justi

Institut für techn. Physik der T.H. Braunschweig (Z. Naturforschg. 4 a, 561-562 [1949]; eingeg. am 27. Okt. 1949)

Die Platinmetalle bieten infolge ihrer teilweise nicht aufgefüllten inneren Elektronenschalen grund-

* 5. Mitt. von E. J u sti u. Mitarbb. über Hall-EffektMessungen; vgl. a. Physik. Z. 42, 349 [1941] (NbN); ebd. 43, 207 [1942] (Re); Ann. Physik 3, 183 [1948] $(\mathrm{Na}, \mathrm{K}) ; \mathrm{Z}$. Naturforschg. 4 a, 472 [1949]. 